\title{
Some results about multifactor uncertain differential equations with applications to extreme values and time integral
}

\author{
Zhifu Jia ${ }^{1}$, Xinsheng Liu ${ }^{1}$, and Yuncai Yu ${ }^{1}$ \\ ${ }^{1}$ Nanjing University of Aeronautics and Astronautics
}

August 20, 2020

\begin{abstract}
Previous literature has proved that there exists a unique solution about multifactor uncertain differential equation (MUDE for short) when their coefficients satisfy strict global Lipschitz continuous condition. In this paper, firstly, we consider new existence and uniqueness theorem under the weaker local Lipschitz continuous condition. The next, when the coefficients do not satisfy the Lipschitz condition, we just showcase existence theorem under continuous and linear growth conditions. Once more, we establish the inverse uncertainty distributions (IUDs for short) of supremum, infimum and time integral about the uncertain process $\mathrm{Zk}$, meanwhile, we design some numerical algorithms for solving these IUDs. In the end, some numerical examples are presented to verify the effectiveness of algorithms.
\end{abstract}

\section{Hosted file}

els.pdf available at https://authorea.com/users/352452/articles/476710-some-results-aboutmultifactor-uncertain-differential-equations-with-applications-to-extreme-values-and-

time-integral 
figures/1/1-eps-converted-to.pdf 
figures/2/2-eps-converted-to.pdf 
figures/3/3-eps-converted-to.pdf 